\title{
Attentive Tracking Disrupts Feature Binding in Visual Working Memory
}

\author{
Daryl Fougnie ${ }^{1}$ and René Marois ${ }^{1}$ \\ ${ }^{1}$ Department of Psychology, Vanderbilt Vision Research Center, Center for Integrative and \\ Cognitive Neuroscience, Vanderbilt University, Nashville, TN 37203
}

\section{Abstract}

One of the most influential theories in visual cognition proposes that attention is necessary to bind different visual features into coherent object percepts (Treisman \& Gelade, 1980). While considerable evidence supports a role for attention in perceptual feature binding, whether attention plays a similar function in visual working memory (VWM) remains controversial. To test the attentional requirements of VWM feature binding, here we gave participants an attention-demanding multiple object tracking task during the retention interval of a VWM task. Results show that the tracking task disrupted memory for color-shape conjunctions above and beyond any impairment to working memory for object features, and that this impairment was larger when the VWM stimuli were presented at different spatial locations. These results demonstrate that the role of visuospatial attention in feature binding is not unique to perception, but extends to the working memory of these perceptual representations as well.

\begin{abstract}
Visual scenes regularly contain multiple objects, each defined by its unique featural properties such as its color, shape and motion. The richness of this visual information presents a 'binding' problem to the visual system: how does our brain correctly associate features with their proper objects, especially considering that these features are processed in distinct and highly specialized cortical regions (DeYoe \& Van Essen, 1988)? While it is not yet fully understood how features become conjoined into objects during visual perception, one influential proposal is that visuospatial attention may assist in the binding process by selecting an object's location and linking all features at that location to the object (Treisman \& Gelade, 1980; Treisman, 1998; 2006). The finding that subjects may incorrectly bind features of objects together if attention is diverted provides converging evidence for a role of attention in perceiving an integrated visual world (Briand \& Klein, 1987; Prinzmetal, Presti, \& Posner, 1986; Treisman \& Schmidt, 1982; but see Donk, 1999; Navon \& Ehrlich, 1995).

While a considerable amount of work has illuminated the function of attention in visual perception, its role in visual working memory (VWM) is much less understood. Dual-task studies have shown that shifts of attention can interfere with VWM (Awh \& Jonides, 2001; Awh, Jonides, \& Reuter-Lorenz, 1998; Fougnie \& Marois, 2006; Oh \& Kim, 2004; Woodman \& Luck, 2004; but see Woodman, Vogel, \& Luck, 2001), clearly highlighting a role for attention in VWM maintenance. Wheeler and Treisman (2002) have proposed that the role of attention in VWM is not in the storage of object features, but rather in the maintenance of proper bindings between these features. According to this view, visuospatial attention plays a similar role in visual perception and VWM-the formation and maintenance of feature
\end{abstract}

Correspondence from the editor/publisher should be addressed to: Daryl Fougnie, Department of Psychology, Wilson Hall, 11121 st Avenue South, Vanderbilt University, Nashville, TN 37240-1103, 615-322-0173 (telephone), 615-343-8449 (fax), E-mail: d.fougnie@vanderbilt.edu (e-mail).. 
bindings. This theory is supported by work suggesting that attention is involved in creating a representational format, termed 'object files', which maintain an object's identity in both perception and working memory (Irwin, 1992, 2002; Irwin \& Andrews, 1996; Irwin \& Zelinsky, 1996; Kahneman \& Treisman, 1984; Kahneman, Treisman, \& Gibbs, 1992; Treisman, 2006; Treisman and Zhang, 2006). In contrast to the view that attention is necessary to maintain feature bindings in VWM, it has been suggested that features are automatically conjoined in VWM. According to this hypothesis, participants can store the same number of items in VWM regardless of whether the items are defined by a single feature, or by a conjunction of features (Luck \& Vogel, 1997; Vogel, Woodman, \& Luck, 2001). Thus, theories of VWM differ on the purported role of attention in maintaining bound features.

If attention is required to keep features bound together in VWM, then diverting attention during a VWM retention interval should result in impaired memory for feature bindings. Indeed, the importance of attention in perceptual feature binding is often demonstrated by the improper feature conjunctions that result when attention is diverted from the primary perceptual task (Treisman \& Schmidt, 1982). When this approach has been used to investigate the role of attention in working memory, the results have generally failed to demonstrate that attention is specifically involved in VWM feature binding (Allen, Baddeley, \& Hitch, 2006; Gajewski \& Brockmole, 2006; Yeh, Yang, \& Chiu, 2005). One study disrupted attention by requiring subjects to identify letters that were briefly presented at various positions during the retention interval of a VWM task (Yeh et al., 2005), while another used a briefly presented exogenous cue to draw attention during the VWM retention interval (Gajewski \& Brockmole, 2006). While both studies showed that the withdrawal of attention affected VWM performance, neither found that it specifically disrupted VWM feature bindings. However, considering that attention may not need to be continuously applied to maintain object representations in VWM (Hollingworth, 2004; Hollingworth, Williams, \& Henderson, 2001; Irwin, 1992, 1996;

Schneider, 1999), transient shifts of attention may be insufficient to impair VWM feature binding. In contrast to the two aforementioned studies, Allen and colleagues (Allen, et al., 2006) used a continuously demanding attention task during the VWM retention interval, but nevertheless still failed to show specific disruptions of VWM feature bindings. However, that study's attention-demanding secondary task - which consisted in either backward counting or supra-span digit recall - may not have tapped onto the type of attention that is required for binding. Backward counting and digit recall tasks are considered to load on central attention (Han \& Kim, 2004) while visuospatial attention is believed to be involved in feature binding during perception (Treisman \& Gelade, 1980), and these two types of attention have been proposed to be distinct (Johnston, McCann, Remington, 1995; Pashler 1991, 1993; Posner \& Petersen, 1990; Vogel, Woodman, \& Luck, 2005).

Thus, the goal of the present study was to re-examine the role of attention in VWM feature binding. Specifically, we tested the hypothesis that a secondary attention task, which is both visuospatial and continuously demanding, could disrupt feature bindings in VWM.

\section{Experiment One}

To test whether a continuously demanding attention task would disrupt feature binding in VWM, we required subjects to perform a multiple object tracking (MOT) task (Cavanagh \& Alvarez, 2005; Pylyshyn \& Storm, 1988) during the retention interval of a VWM task (Figure 1). The MOT task involved attentively tracking moving targets among distractors. The VWM task involved holding in working memory, over a brief retention interval, just the color, just the shape, the color and the shape, or the specific conjunctions of color and shape of the objects in the memory display. Based on previous work (Fougnie \& Marois, 2006), we predicted that the MOT task would disrupt VWM performance. More importantly, we predicted that the MOT 
task would be most disruptive for VWM in the conjunction condition because this condition should specifically rely on the type of attention taxed by MOT.

\section{Methods}

Participants-Twenty young adults ( 8 males) participated for course credit or monetary reward. One participant's data was discarded because VWM performance did not meet our $a$ priori criterion of above chance VWM performance in all conditions. This criterion prevented the inclusion of participants who would not show an effect of tracking on VWM performance because of chance VWM performance even without the tracking task.

\section{Procedure}

VWM task: This task was based on a change detection procedure (Vogel, et al., 2001) in which a memory display of three objects was first briefly presented, followed by a retention interval, and by a single probe item that required a same-different judgment on the task-relevant feature (s). The relevant features differed across four VWM conditions (Color-only, Shape-only, Either, Binding). In the Color-only condition, participants memorized the color of the stimuli, whereas they memorized the shape in the Shape-only condition. In Either and Binding conditions, participants were required to memorize both the color and shape of the stimuli, but only the Binding condition required participants to remember the specific color-shape pairings of the objects in the memory display.

The memory display consisted of three colored shapes $\left(0.78^{\circ} \times 0.78^{\circ}\right)$. Stimuli were spaced evenly apart and appeared along an imaginary circle $\left(2.5^{\circ}\right.$ radius $)$ around a centrally presented fixation cross. There were six possible colors (green, brown, blue, yellow, purple, or red) and six possible shapes (circle, square, octagon, cross, diamond, or triangle). Task-relevant features did not repeat in a display. In the Color-only condition, the stimuli all shared the same shape, selected randomly every trial. Similarly, the stimuli all shared the same randomly selected color in the Shape-only condition.

The memory display was presented for $400 \mathrm{~ms}$ and, after a $6800 \mathrm{~ms}$ retention interval, a probe display appeared containing one colored shape $\left(0.78^{\circ} \times 0.78^{\circ}\right)$ at fixation. The probe display remained until participants made an unspeeded response (accuracy stressed) by pressing one of two keys on a keyboard with their right hand to indicate whether the task-relevant features matched an item from the memory display (50\% match trials). In the Color-only and Shapeonly conditions, the probe either matched one of the memory items, or differed in the taskrelevant feature. In the Either condition, the probe display tested shape or color memory (randomly determined). To minimize confusion in this condition, the untested feature property was a color (black) or shape (bar) that would never appear in the memory display (see Figure 2). In the binding condition, the probe was either a correct or incorrect pairing of shape and color from the memory display.

MOT task: The MOT task occurred during the VWM retention interval beginning $800 \mathrm{~ms}$ after memory display offset to allow sufficient time to fully encode the VWM display. MOT stimuli were hollow white discs $\left(0.50^{\circ}\right)$ moving about within an $8.5^{\circ} \times 8.5^{\circ}$ area centered around fixation. Each $6,000 \mathrm{~ms}$ MOT movie had three phases: marker, tracking, and probe, lasting $2,000,3,000$, and 1,000ms respectively. During the marker phase, the three target discs (among nine distractors) were filled white. During the tracking phase, the target indicators disappeared and both targets and distractors began to move in a random direction. The speed of each disc varied from $0.00215^{\circ} / \mathrm{ms}$ to $0.0195 \% \mathrm{~ms}$, and disc direction had a $10 \%$ chance to change at every refresh (13.3ms). The discs bounced off the boundaries of the MOT display, other MOT discs, and the three VWM stimuli locations. During the probe phase, a single disc was marked. Half of the time the probe disc corresponded to a target. 
Participants responded to the tracking task after responding to the VWM task. A question mark appeared above fixation, notifying participants to make a left-handed button press to indicate whether the MOT probe item corresponded to a target. Responses were not speeded and accuracy was stressed.

Control condition: Performance in the VWM task during concurrent MOT was contrasted with VWM performance during a Control condition in which a stimulus display similar to that of the MOT task was presented but no accompanying task was required. The Control condition included a MOT display during the VWM retention interval, as in the MOT task, except that no targets were highlighted in the marker phase and all discs were highlighted during the probe phase. In the Control condition, only the VWM response was collected.

Articulatory suppression: To minimize contamination form verbal working memory, participants performed an articulatory suppression task (repeating the word 'the' at a 2-HZ rate) beginning one second prior to the memory display and continuing until responses for both tasks had been collected. Articulatory suppression was monitored by an experimenter.

Design: Participants performed eight blocks of 24 trials. Half of the trials in each block required tracking, with the tracking and control trials randomly intermixed. VWM conditions were blocked, and participants were assigned to one of four possible orderings, with each ordering differing by the starting condition. For each ordering, the four conditions were first presented sequentially for the first four blocks, and this sequence was reversed for the last four blocks (for example, a participant might receive Either-Binding-Color-Shape-Shape-Color-BindingEither blocks of trials). In all experiments, there was no effect of VWM condition order on either VWM or MOT performance $(p ' s$ ' .5$)$, thus this factor is not discussed any further. At the beginning of the experiment participants completed a practice block consisting of 12 trials of each VWM type. Participants were instructed to maintain fixation on the central cross throughout the trial and to emphasize the two tasks equally.

\section{Results}

VWM task-VWM accuracy is plotted in Figure 3A. Following prior convention, we separately analyzed color and shape probes in the Either condition (labeled Either-Color and Either-Shape respectively; see also Allen, et al., 2006; Wheeler \& Treisman, 2002).

Importantly, the results of this experiment (and Experiment 2) are statistically equivalent if the analyses are performed on the combined Either condition data. We performed a two-way within-subject ANOVA as a function of tracking condition (Tracking, Control) and VWM condition (Color-only, Shape-only, Either-Color, Either-Shape, Binding) on the VWM accuracy data. There were main effects of VWM condition, $F(4,72)=31.3, p<.0001$, and tracking condition, $F(1,18)=13.9, p<.005$. The interaction between tracking and VWM condition was also significant, $F(4,72)=5.3, p<.01$. To measure the effect of tracking in each VWM condition, VWM accuracy during no tracking (Control condition) was subtracted from VWM accuracy during tracking. Two-tailed $t$-tests revealed an effect of tracking in the Binding $(p<.001)$ and Shape-only conditions $(p=.02)$, but not in any other VWM conditions (all, $p ' s>.05)$. Most importantly, two-tailed paired $t$-tests demonstrated that the effect of tracking was significantly larger in the Binding condition than in any other VWM condition, all $p$ 's $<$. 05.

MOT task-MOT accuracy is illustrated in Figure 3B. A one way within-subject ANOVA, with a factor of VWM condition (Color-only, Shape-only, Either-Color, Either-Shape, Binding), revealed that VWM condition had no affect on MOT performance, $F(4,72)=1.02$, $p=.41$. 


\section{Discussion}

An attention-demanding tracking task performed during the retention interval of a VWM task specifically disrupted feature bindings in VWM. That the effect of tracking was largest in the Binding condition cannot be explained by an unequal allocation of resources to tracking between VWM conditions, since performance on the tracking task was comparable across these conditions. This result also cannot be explained by the fact that tracking can additively impair VWM performance of two features in the Binding condition compared to a single feature in the Color-only and Shape-only conditions because the costs of tracking in the Binding condition are larger than the sum of the costs of tracking in the Color-only and Shape-only conditions ( $\mathrm{p}<.05$, one tailed). It is also unlikely that the larger Binding interference occurred because that condition was more difficult than the other conditions. While VWM performance in the absence of tracking was worse in the Binding condition than either the Color-only or Shape-only condition $\left(p^{\prime} s<.001\right)$, it was equivalent in the Binding and Either conditions $(p$ $=.88$ ). The latter results accord well with those of Allen and colleagues (2006) demonstrating that general secondary task difficulty does not affect VWM binding.

Taken together, the findings of Experiment 1 are consistent with Wheeler and Treisman's (2002) suggestion that disruptions of visuospatial attention impair VWM binding. However, in addition to attentive tracking, the MOT task involves other cognitive processes, including encoding and retrieval of target identity. In order to demonstrate that the interference of MOT on VWM feature binding results from attentional demands, non-attentional factors need to be ruled out. Experiment 2 accomplished this by replacing the Control task of Experiment 1 with a Static task. The Static task was identical to the Tracking task during the marker and probe phases, but the tracking phase differed in that the stimuli remained stationary instead of moving about throughout that phase. Although the Static condition still necessitated the encoding and retrieval of targets, the requirement to attentively track stimuli was virtually eliminated. If the impairment in Experiment 1 was due to the demands of tracking, then binding should still be impaired during tracking relative to the Static condition.

\section{Experiment Two}

\section{Method}

Participants-Twenty-five young adults (11 males) participated for course credit or monetary reward. Data from three participants were discarded because of chance performance in one of the VWM conditions.

Procedure-The procedure for Experiment 2 was identical to Experiment 1 except for the following differences.

VWM task: The color (green, brown, red, yellow and blue) and shape (triangle, octagon, cross, diamond, and square) sets were altered to further minimize potential confusion between tasks.

Static memory task: The Static task had three phases: marker (2s), memory (3s), and probe (1s). The memory phase was identical to the tracking phase of the MOT task, except that the disks remained stationary.

A total of 256 trials were included with 32 trials in each block. The type of probe in the Either condition was counterbalanced across conditions.

\section{Results and Discussion}

VWM task-VWM accuracy is plotted in Figure 4A. We performed a two-way within-subject ANOVA as a function of tracking condition (Tracking, Static) and VWM condition (Color- 
only, Shape-only, Either-Color, Either-Shape, Binding) on VWM accuracy. There was a main effect of VWM condition, $F(4,84)=71.9, p<.0001$, and tracking condition, $F(1,21)=6.6, p$ $<.05$, as well as an interaction between tracking and VWM condition, $F(4,84)=2.4, p<.05$. Two-tailed $t$-tests showed an effect of tracking (VWM accuracy during the Static condition subtracted from VWM accuracy during tracking) in the Binding condition $(p<.001)$ but not in any other VWM condition ( $p$ 's > .05). Additionally, two-tailed, paired t-tests revealed that the effect of tracking was larger in the Binding condition than in all other conditions (Coloronly, Shape-only, Either-Color, Either-Shape), all $p$ ' $s .05$. Furthermore, costs due to tracking were larger in the Binding condition than the added costs of tracking in the Color-only and Shape-only conditions $(p=.01)$. The observed impairment in VWM performance with concurrent tracking, which was largest in the Binding condition, replicates Experiment 1's findings while ruling out the possibility that non-attentional components of the MOT task (i.e. encoding and retrieval) caused the binding impairment.

Secondary task-Secondary task accuracy is plotted in Figure 4B. We performed a twoway within-subject ANOVA as a function of tracking condition (Tracking, Static) and VWM condition (Color-only, Shape-only, Either-Color, Either-Shape, Binding) on secondary task accuracy. There was a main effect of VWM condition, $F(4,84)=2.9, p<.05$, and tracking condition, $F(1,21)=89.3, p<.001$ (Tracking worse than Static), but no interaction between tracking and VWM condition, $F(4,84)=.7, p=.6$. Consistent with the failure to find an interaction between tracking and VWM condition, paired t-tests revealed that the difference in secondary task accuracy (Tracking minus Static) in the Binding condition was similar to the other VWM conditions ( $p^{\prime}$ s > .2). Thus, the large interference of tracking on VWM performance in the Binding condition cannot be explained by participants differentially allocating resources to the VWM and secondary tasks across conditions.

\section{Experiment Three}

It has been suggested that visuospatial attention promotes feature binding during visual perception by disambiguating the relationship between features and objects in a visual scene containing multiple objects (Desimone \& Duncan, 1995; Luck \& Ford, 1998; Luck, Girelli, McDermott, \& Ford, 1997; Treisman, 1998, 2006). According to this proposal, attention selects an object location and filters out all other unattended objects, allowing the attended features to be bound together into an object representation (Treisman, 1998, 2006). Consistent with this disambiguation theory, there is both neurological (Friedman-Hill, Robertson, \& Treisman, 1995) and neuroimaging (Shafritz, Gore, \& Marois, 2002) evidence that the posterior parietal cortex - a brain region critical for visuospatial attention (Kastner \& Ungerleider, 2000; Shafritz et al., 2002) - promotes feature binding chiefly when there is potential for confusion about the relationship between each object and their constituent features. Specifically, the posterior parietal cortex was found to be preferentially involved in feature integration when multiple objects were presented simultaneously in a visual display, but not when they were presented sequentially at the same location (Friedman-Hill et al., 2005; Shafritz et al., 2002). Following on these findings, we tested in the present experiment whether the role of visuospatial attention in maintaining feature bindings would be reduced if the VWM stimuli were presented sequentially at fixation. If so, this would suggest that one role of attention in VWM is the maintenance of location-specific object representations formed during visual perception (Treisman \& Zhang, 2006).

\section{Method}

Participants-Twenty-five young adults (11 males) participated for course credit or monetary reward. One participant's data was removed from all analyses because she was not engaging in articulatory suppression. 
Procedure-The procedure for Experiment 3 was identical to Experiment 2 except that the VWM stimuli were presented sequentially at fixation. Each stimulus was presented for $400 \mathrm{~ms}$ with a 400ms inter-stimulus interval. The Color and Shape conditions were not included since the principal comparison is between the Binding and Either conditions.

\section{Results and Discussion}

VWM task-VWM accuracy is plotted in Figure 5A. We performed a two-way within-subject ANOVA as a function of tracking condition (Tracking, Static) and VWM condition (EitherColor, Either-Shape, Binding) on the VWM accuracy data. There was a main effect of VWM condition, $F(2,46)=53.78, p<.0001$, but there was no effect of tracking condition, $F(1,23)$ $=.04, p=.84$, and a marginal interaction between tracking and VWM condition, $F(2,36)=$ 2.94, $p=.06$. A two-tailed $t$-test revealed a marginal effect of tracking in the Binding condition (VWM performance during the Static condition subtracted from VWM performance during the Tracking condition, $p=.07$ ). The effect of tracking in the Binding condition was similar to the one observed in the Either-Color or Either-Shape conditions (two-tailed paired $t$-tests, both $p$ 's > .15). However, we did observe a difference in the effect of tracking between the Binding and the combined Either condition $(p=.04)$, suggesting that tracking might still impair feature binding in VWM even when VWM stimuli are presented sequentially at fixation. Importantly, this effect is much reduced compared to when VWM stimuli were presented at distinct locations in Experiment 2 (two-sample $t$-test of the tracking effect in the Binding condition between Experiments 2 and 3, $p<.05$; Figures $4 \& 5$ ).

Previous studies have found that visuospatial attention is primarily involved in perceptual feature binding when objects are presented at distinct spatial locations (Friedman-Hill, et al., 1995; Shafritz, et al., 2002). Correspondingly, here we show that the amount of impairment produced by a secondary tracking task on VWM feature binding was significantly reduced when the VWM stimuli were presented sequentially at the same location. However, although the tracking effect was attenuated it was not altogether eliminated with foveal stimulus presentation. These results suggest that multiple object tracking may disrupt VWM feature binding by affecting both spatial and non-spatial attentional components of working memory.

Secondary task-We performed a two-way within-subject ANOVA as a function of tracking condition (Tracking, Static) and VWM condition (Either-Color, Either-Shape, Binding) on secondary task accuracy (Figure 5B). The main effect of tracking condition was significant, $F(1,23)=16.5, p<.001$, but there was no main effect of VWM condition, $F(2,46)$ $=2.25, p=.12$, and no interaction between tracking and VWM condition, $(2,46)=.99, p=$. 38. Paired t-tests revealed that the difference in secondary task accuracy (Tracking minus Static) in the Binding condition was similar to the other VWM conditions ( $p$ 's > .2), suggesting that the failure to observe significant effects of tracking on VWM performance cannot be explained by participants differentially allocating resources to the VWM and secondary tasks across conditions.

\section{General Discussion}

In the present study, we carried out three experiments to test the hypothesis that visuospatial attention supports feature binding in visual working memory. In Experiment 1 we found that a secondary tracking task impaired memory for color-shape bindings to a larger degree than memory for single features such as color or shape. Experiment 2 showed that the effect is due to the attentive tracking component of the tracking task, and Experiment 3 demonstrated that the interference of the MOT task on VWM feature binding performance largely depends on the VWM stimuli occupying distinct spatial locations. Taken together, the results of these three experiments support the hypothesis that visuospatial attention plays an important role in storing 
bound representations in VWM (Delvenne \& Bruyer, 2004; Treisman \& Wheeler, 2002). These findings are consistent with a study suggesting that VWM feature binding is vulnerable to the spatial configuration of objects in a visual display (Treisman and Zhang, 2006), and with neuroimaging studies showing that brain regions involved in visuospatial attention and VWM - particularly the parietal cortex - also support visual feature binding (Corbetta, et al., 2000; Corbetta, Shulman, Miezin, \& Petersen, 1995; Culham, Cavanagh, \& Kanwisher, 2001; Jovicich et al., 2001; Shafritz et al., 2002; Todd \& Marois, 2004).

Our results are at variance with previous studies that did not observe an effect of a secondary task on VWM feature binding (Allen et al., 2006; Gajewski \& Brockmole, 2006; Yeh et al., 2005). This difference may be attributed to the fact that some of these studies used secondary tasks that loaded on central attention rather than on visuospatial attention. Central attention may not contribute to VWM binding, even when the secondary central tasks (e.g. digit recall) are just as difficult as ours (Allen, et al., 2006). Consistent with this notion, we found in Experiment 3 that a secondary task that puts heavy demands on spatial attention barely affected VWM feature binding when spatial information could no longer be used for disambiguating the relationship between objects and features in VWM. Additionally, studies that did not use continuously demanding secondary tasks may not have observed VWM binding-specific deficits because the secondary task may not have sufficiently taxed attention (Gajewski \& Brockmole, 2006; Yeh et al., 2005). A similar argument may also account for the difference between our results and those of a recent study (Johnson, Hollingworth, \& Luck, 2008) reporting that a visual search task does not disrupt feature bindings in VWM. Because the visual search task demanded attention for a shorter duration $(\approx 1 \mathrm{~s})$ than our MOT task $(6 \mathrm{~s})$ and may only have required few shifts of visuospatial attention (two on average for set sizes of four), that task may have insufficiently taxed attentional resources to specifically impair feature bindings in VWM.

Previous work (Desimone \& Duncan, 1995; Friedman-Hill et al., 1995; Shafritz, et al., 2002) has suggested that visuospatial attention can be used to create bound object representations if objects occur at distinct spatial locations. Our dual-task study demonstrates that visuospatial attention also appears critical for the continued maintenance of these representations. In conjunction with previous findings, this may suggest that attention plays a critical role in VWM when spatial information is important for the VWM task (Awh, et al., 1998; Awh \& Jonides, 2001; Oh \& Kim, 2004; Treisman \& Zhang, 2006; Woodman \& Luck, 2004; Woodman, Vogel, \& Luck, 2001). In analogy to the proposed role of visuospatial attention in spatial working memory (Awh, et al., 1998; Awh \& Jonides, 2001; Awh, Vogel, \& Oh, 2006; Postle, Awh, Jonides, Smith, \& D'Esposito, 2004; Smith \& Jonides, 1998; Smyth \& Scholey, 1994), visuospatial attention may assist VWM by scanning the objects' locations during the retention interval as a means of refreshing the features that are bound to that location. If the role of attention in VWM primarily consists in iteratively refreshing stored representations, it could also explain why bound VWM representations may only be impaired by a secondary task that strongly and continuously withdraws attention away from working memory.

While the present results suggest a role for visuospatial attention in feature binding in VWM, they do not imply that such binding is solely dependent on visuospatial attention. Indeed, it is interesting to note that, even with a concurrent difficult tracking task, participants' VWM performance was nevertheless above chance in the Binding condition (50\% being chance performance in Figs. 3-5). These results are consistent with the notion that some binding can occur in the absence of visuospatial attention (Holcombe \& Cavanagh, 2001). It is also consistent with the recent finding that VWM capacity, including the capacity for color-location bindings, is not solely based on visuospatial attention, but is also constrained by central attention and VWM-specific sources of processing (Fougnie \& Marois, 2006). But irrespective of the extent to which other factors may contribute to feature binding in VWM, the present 
results suggest that visual features are not automatically integrated in VWM. Instead, working memory of feature bindings, just as their perceptual representations, is seemingly vulnerable to the withdrawal of visuospatial attention.

\section{Acknowledgements}

This work was supported by NIMH grant (R01-MH70776) to R.M.

\section{References}

Allen RJ, Baddeley AD, Hitch GJ. Is the binding of visual features in working memory resourcedemanding? Journal of Experimental Psychology: General 2006;135(2):298-313. [PubMed: 16719655]

Awh E, Jonides J. Overlapping mechanisms of attention and spatial working memory. Trends in Cognitive Sciences 2001;5:119-126. [PubMed: 11239812]

Awh E, Jonides J, Reuter Lorenz PA. Rehearsal in spatial working memory. Journal of Experimental Psychology: Human Perception and Performance 1998;24:780-790. [PubMed: 9627416]

Awh E, Vogel EK, Oh SH. Interactions between attention and working memory. Neuroscience 2006;139 (1):201-208. [PubMed: 16324792]

Briand KA, Klein RM. Is posner's "beam" the same as treisman's "glue?" on the relation between visual orienting and feature integration theory. Journal of Experimental Psychology: Human Perception \& Performance 1987;13(2):228-241. [PubMed: 2953853]

Cavanagh P, Alvarez GA. Tracking multiple targets with multifocal attention. Trends in Cognitive Sciences 2005;9(7):349-354. [PubMed: 15953754]

Corbetta M, Kincade JM, Ollinger JM, McAvoy MP, Shulman GL. Voluntary orienting is dissociated from target detection in human posterior parietal cortex. Nature Neuroscience 2000;3(3):292-297.

Corbetta M, Shulman GL, Miezin FM, Petersen SE. Superior parietal cortex activation during spatial attention shifts and visual feature conjunction. Science 1995;270(5237):802-805. [PubMed: 7481770]

Culham JC, Cavanagh P, Kanwisher NG. Attention response functions: Characterizing brain areas using fmri activation during parametric variations of attentional load. Neuron 2001;32(4):737-745. [PubMed: 11719212]

Delvenne JF, Bruyer R. Does visual short-term memory store bound features? Visual Cognition 2004;11 (1):1-27.

Desimone R, Duncan J. Neural mechanisms of selective visual attention. Annual Review of Neuroscience 1995;18:193-222.

DeYoe EA, Van Essen DC. Concurrent processing streams in monkey visual cortex. Trends in Neurosciences 1988;11(5):219-226. [PubMed: 2471327]

Donk M. Illusory conjunctions are an illusion: The effects of target-nontarget similarity on conjunction and feature errors. Journal of Experimental Psychology: Human Perception and Performance 1999;25 (5):1207-1233.

Fougnie D, Marois R. Distinct capacity limits for attention and working memory: Evidence from attentive tracking and visual working memory paradigms. Psychological Science 2006;17(6):526-534. [PubMed: 16771804]

Friedman-Hill SR, Robertson LC, Treisman A. Parietal contributions to visual feature binding: Evidence from a patient with bilateral lesions. Science 1995;269(5225):853-855. [PubMed: 7638604]

Gajewski DA, Brockmole JR. Feature bindings endure without attention: Evidence from an explicit recall task. Psychonomic Bulletin and Review 2006;13(4):581-587. [PubMed: 17201355]

Han SH, Kim MS. Visual search does not remain efficient when executive working memory is working. Psychological Science 2004;15(9):623-628. [PubMed: 15327634]

Holcombe AO, Cavanagh P. Early binding of feature pairs for visual perception. Nature Neuroscience 2001;4(2):127-128.

Hollingworth A. Constructing visual representations of natural scenes: The roles of short- and long-term visual memory. Journal of Experimental Psychology: Human Perception and Performance 2004;30 (3):519-537. [PubMed: 15161384] 
Hollingworth A, Williams CC, Henderson JM. To see and remember: Visually specific information is retained in memory from previously attended objects in natural scenes. Psychonomic Bulletin and Review 2001;8(4):761-768. [PubMed: 11848597]

Irwin DE. Memory for position and identity across eye movements. Journal of Experimental Psychology: Learning, Memory, \& Cognition 1992;18(2):307-317.

Irwin DE. Integrating information across saccadic eye movements. Current Directions in Psychological Science 1996;5(3):94-100.

Irwin, DE.; Andrews, RV., editors. Integration and accumulation of information across saccadic eye movements. The MIT Press; Cambridge, MA, US: 1996.

Irwin DE, Zelinsky GJ. Eye movements and scene perception: Memory for things observed. Perception and Psychophysics 2002;64(6):882-895. [PubMed: 12269296]

Johnston JC, McCann RS, Remington RW. Chronometric evidence for two types of attention. Psychological Science 1995;6(6):365-369.

Johnson JS, Hollingworth A, Luck SJ. The role of attention in the maintenance of feature bindings in visual short-term memory. Journal of Experimental Psychology: Human Perception \& Performance 2008;4(1):41-55. [PubMed: 18248139]

Jovicich J, Peters RJ, Koch C, Braun J, Chang L, Ernst T. Brain areas specific for attentional load in a motion-tracking task. Journal of Cognitive Neuroscience 2001;13(8):1048-1058. [PubMed: 11784443]

Kahneman, D.; Treisman, A. Changing views of attention and automaticity. In: Parasuraman, R.; Davies, DR., editors. Varieties of attention. Academic Press; Orlando, FA: 1984. p. 29-61.

Kahneman D, Treisman A, Gibbs BJ. The reviewing of object files: Object-specific integration of information. Cognitive Psychology 1992;24:175-219. [PubMed: 1582172]

Kastner S, Ungerleider LG. Mechanisms of visual attention in the human cortex. Annual Review of Neuroscience 2000;23:315-341.

Luck SJ, Ford MA. On the role of selective attention in visual perception. Proceedings of the National Academy of Sciences of the United States of America 1998;95(3):825-830. [PubMed: 9448247]

Luck SJ, Girelli M, McDermott MT, Ford MA. Bridging the gap between monkey neurophysiology and human perception: An ambiguity resolution theory of visual selective attention. Cognitive Psychology 1997;33(1):64-87. [PubMed: 9212722]

Luck SJ, Vogel EK. The capacity of visual working memory for features and conjunctions. Nature 1997;390(6657):279-281. [PubMed: 9384378]

Navon D, Ehrlich B. Illusory conjunctions: Does inattention really matter? Cognitive Psychology 1995;29 (1):59-83.

Oh SH, Kim MS. The role of spatial working memory in visual search efficiency. Psychonomic Bulletin and Review 2004;11(2):275-281. [PubMed: 15260193]

Pashler H. Shifting visual attention and selecting motor responses: Distinct attentional mechanisms. Journal of Experimental Psychology: Human Perception and Performance 1991;17(4):1023-1040. [PubMed: 1837295]

Pashler, H., editor. Dual-task interference and elementary mental mechanisms. The MIT Press; Cambridge, MA, US: 1993.

Posner MI, Petersen SE. The attention system of the human brain. Annual Review of Neuroscience 1990;13:25-42.1990

Postle BR, Awh E, Jonides J, Smith EE, D'Esposito M. The where and how of attention-based rehearsal in spatial working memory. Cognitive Brain Research 2004;20(2):194-205. [PubMed: 15183391]

Prinzmetal W, Presti DE, Posner MI. Does attention affect visual feature integration? Journal of Experimental Psychology: Human Perception \& Performance 1986;12(3):361-369. [PubMed: 2943864]

Pylyshyn ZW, Storm RW. Tracking multiple independent targets: Evidence for a parallel tracking mechanism. Spatial Vision 1988;3(3):179-197. [PubMed: 3153671]

Shafritz KM, Gore JC, Marois R. The role of the parietal cortex in visual feature binding. Proceedings of the National Academy of Science, USA 2002;99:10917-10922. 
Smith EE, Jonides J. Neuroimaging analyses of human working memory. Proceedings of the National Academy of Sciences of the United States of America 1998;95(20):12061-12068. [PubMed: 9751790]

Smyth MM, Scholey KA. Interference in immediate spatial memory. Memory and Cognition 1994;22 (1):1-13.

Schneider WX. Visual-spatial working memory, attention, and scene representation: A neuro-cognitive theory. Psychological Research 1999;62(23):220-236. [PubMed: 10472201]

Todd JJ, Marois R. Capacity limit of visual short-term memory in human posterior parietal cortex. Nature 2004;428:751-754. [PubMed: 15085133]

Treisman A. Feature binding, attention and object perception. Philosophical Transactions of the Royal Society of London - Series B: Biological Sciences 1998;353(1373):1295-1306.

Treisman A. How the deployment of attention determines what we see. Visual Cognition 2006;14(48): 411-443. [PubMed: 17387378]

Treisman A, Gelade G. A feature-integration theory of attention. Cognitive Psychology 1980;12(1):97136. [PubMed: 7351125]

Treisman A, Schmidt H. Illusory conjunctions in the perception of objects. Cognitive Psychology 1982;14 (1):107-141. [PubMed: 7053925]

Treisman A, Zhang W. Location and binding in visual working memory. Memory and Cognition 2006;34 (8):1704-1719.

Vogel EK, Woodman GF, Luck SJ. Storage of features, conjunctions, and objects in visual working memory. Journal of Experimental Psychology: Human Perception and Performance 2001;27(1):92114. [PubMed: 11248943]

Vogel EK, Woodman GF, Luck SJ. Pushing around the locus of selection: Evidence for the flexibleselection hypothesis. Journal of Cognitive Neuroscience 2005;17(12):1907-1922. [PubMed: 16356328]

Wheeler ME, Treisman AM. Binding in short-term visual memory. Journal of Experimental Psychology: General 2002;131(1):48-64. [PubMed: 11900102]

Woodman GF, Luck SJ. Visual search is slowed when visuospatial working memory is occupied. Psychonomic Bulletin and Review 2004;11(2):269-274. [PubMed: 15260192]

Woodman GF, Vogel EK, Luck SJ. Visual search remains efficient when visual working memory is full. Psychological Science 2001;12(3):219-224. [PubMed: 11437304]

Yeh YY, Yang CT, Chiu YC. Binding or prioritization: The role of selective attention in visual shortterm memory. Visual Cognition 2005;12(5):759-799. 


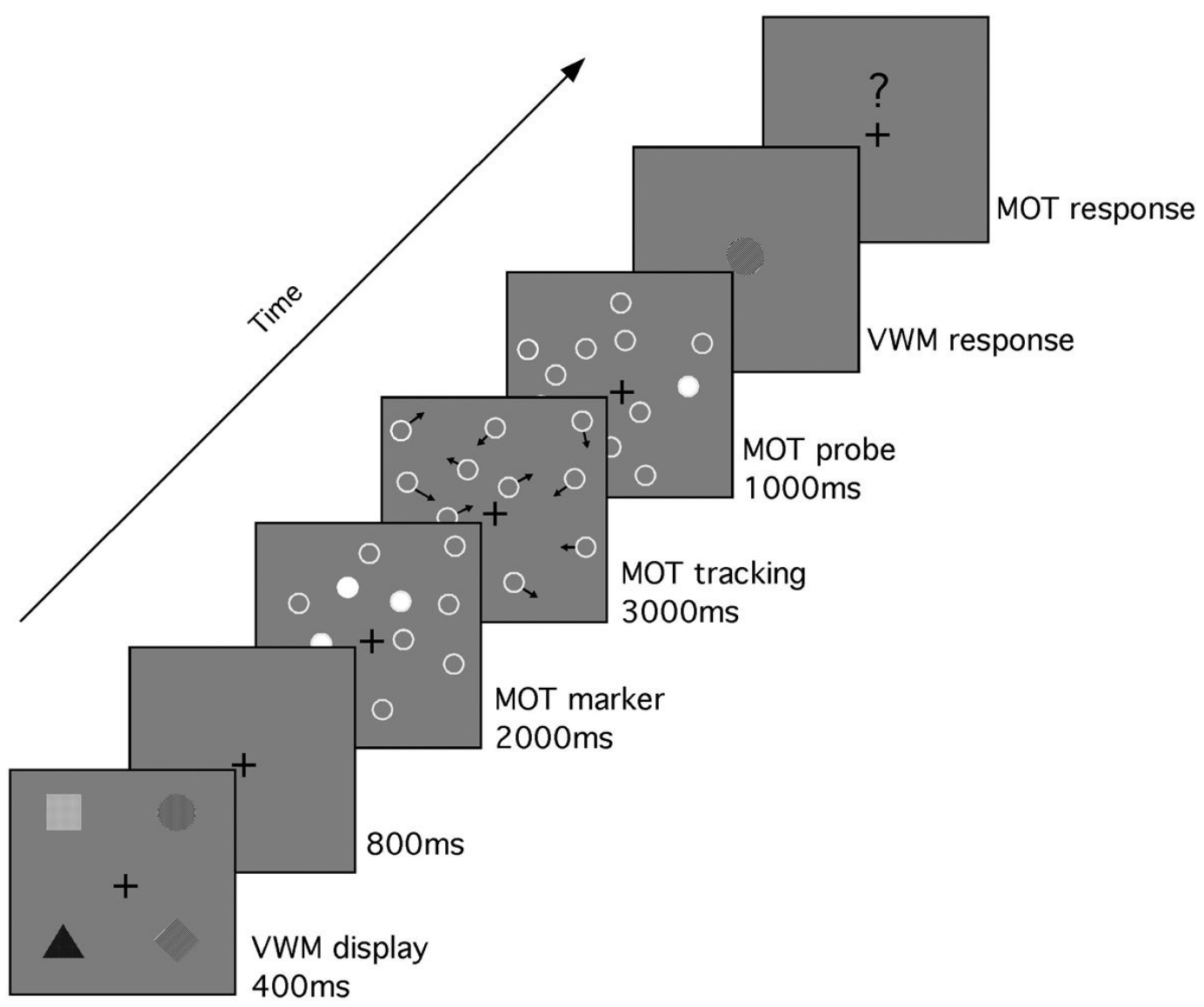

Figure 1.

Trial design for Experiment 1. Different fill patterns represent different solid colors. 


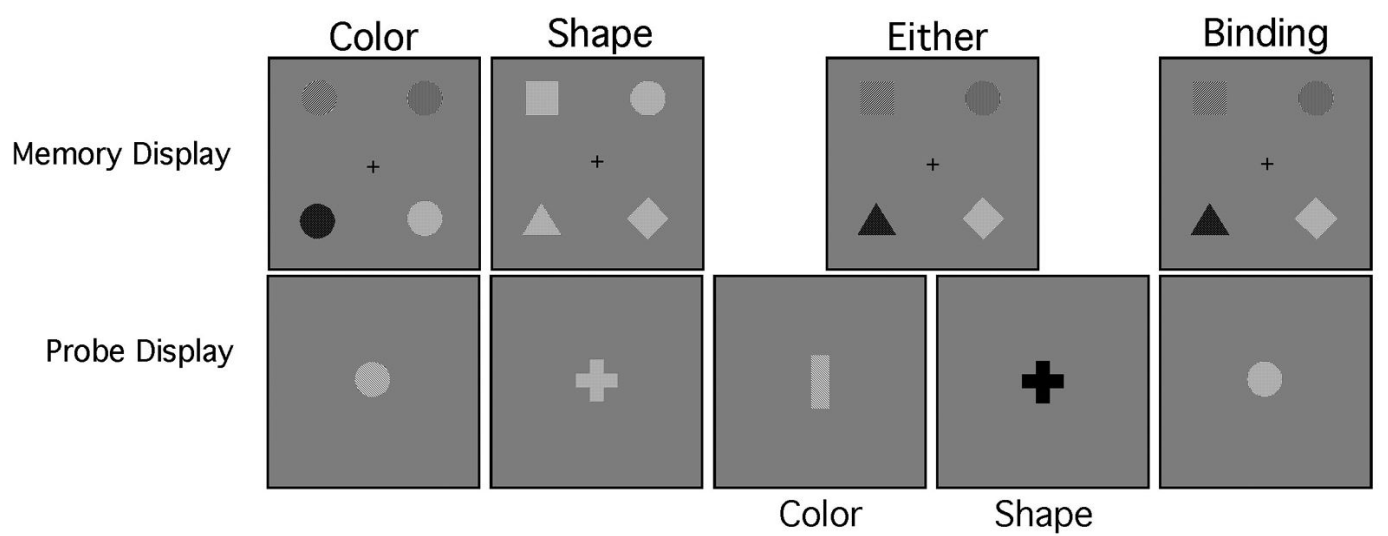

Figure 2.

Examples of memory and probe displays in the four VWM conditions (Color, Shape, Either and Binding). Different fill patterns represent different solid colors. 
a

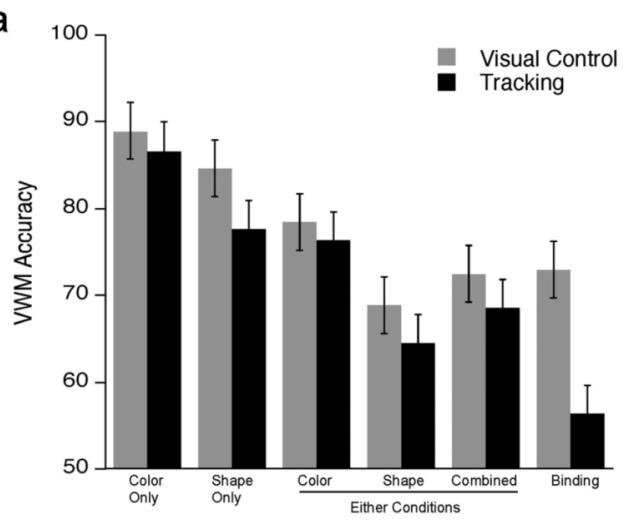

b

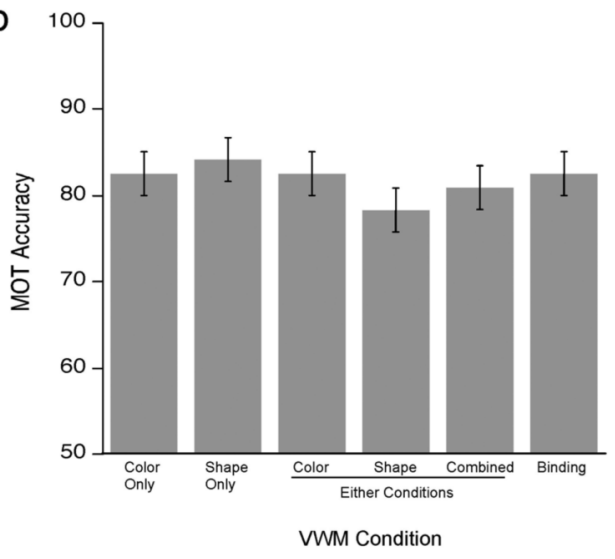

Figure 3.

Accuracy in Experiment 1 for the VWM task (a) and the MOT task (b) by VWM condition. In the Either condition, in addition to combined performance, scores are separately given for color and shape memory probes. VWM accuracy (a) is black for the Tracking condition and gray for the Control condition. Error bars represent within-subject errors of the mean. 

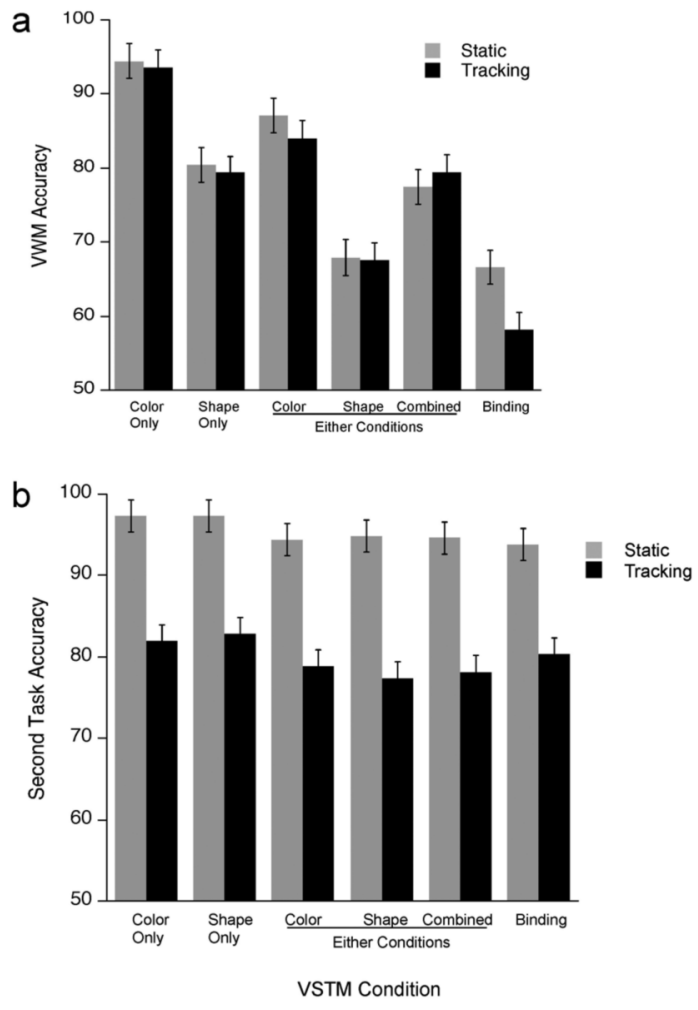

Figure 4.

Accuracy in Experiment 2 for the VWM task (a) and the MOT task (b) by VWM condition. VWM accuracy (a) is black during Tracking and gray during the Static condition. MOT accuracy (b) is black for the Tracking condition and gray for the Static condition. Error bars represent within-subject errors of the mean. 
a
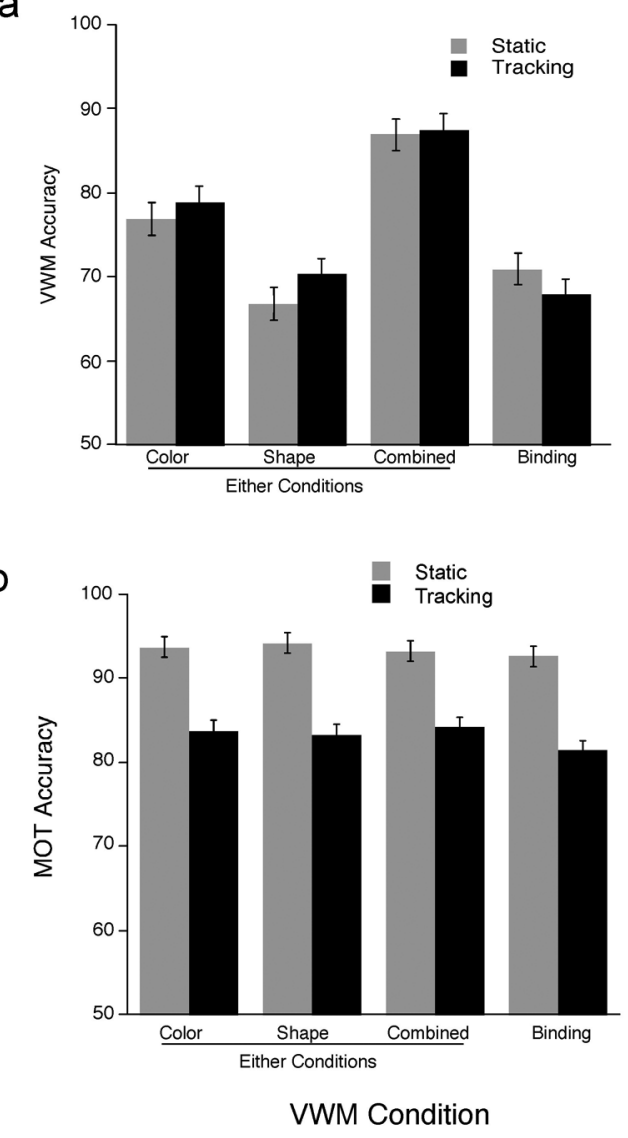

Figure 5.

Accuracy in Experiment 3 for the VWM task (a) and the MOT task (b) by VWM condition. VWM accuracy (a) is black during Tracking and gray during the Static condition. MOT accuracy (b) is black for the Tracking condition and gray for the Static condition. Error bars represent within-subject errors of the mean. 